\title{
The prevalence of child sexual abuse in South Africa: The Optimus Study South Africa
}

The Optimus Study South Africa ${ }^{[1]}$ provides nationally representative data on the extent and impact of child sexual abuse in South Africa (SA)

In this study, 9717 adolescents (ages $15-17$ years) were recruited nationally from schools $(n=4086)$ and households $(n=5$ 631). The definition of sexual abuse used in the study was highly inclusive, including both the range of abuses defined in existing sexual offences laws and those children and adolescents have identified to be sexually intimidating, abusive or exploitative in previous studies (e.g. being forced to view pornography).

The Optimus Study SA shows that sexual abuse of children and adolescents is widespread: $36.8 \%$ of boys and $33.9 \%$ of girls reported some form of sexual abuse. That is, overall, $35.4 \%$ - one in every three adolescents - reported having experienced some form of sexual abuse at some point in their lives.

There were some differences by location and method of data collection, suggesting that the highest disclosure rates (particularly from boys) were obtained from self-completed questionnaires collected in schools. All the children were interviewed and given the opportunity to complete a confidential questionnaire on their own. Of the 4086 young people interviewed in schools, $16.8 \%$ reported experiencing some form of sexual abuse. However, of those same young people who filled out a questionnaire themselves, 35.4\% (or 1399 children and adolescents) reported some form of sexual abuse. Only the household survey allowed us to estimate back to the population. Of the young people interviewed in the household survey, $14.6 \%$ reported some form of sexual abuse; in the general population of adolescents between the ages of 15 and 17, this means that an estimated 454051 have experienced some form of sexual abuse. In the self-completed questionnaire in households, 26.3\% (or 784967 of the youth population) reported experiencing some form of abuse.

Our data also found that boys reported as much risk as girls, a departure from much of the literature. However, there is an important distinction between boys' and girls' experiences of sexual abuse. Girls were more likely to experience forced and penetrative sexual abuse, and other forms of sexual abuse that involve contact with the abuser (contact abuse), while boys were more likely to report forced exposure to sexual acts and material (non-contact abuse).

Experiences of sexual abuse are often not defined by one incident. Lifetime prevalence of abuse may involve abuse by the same person over a period of years or by a number of different people in different contexts. We often think about child abuse as something committed by a single person, a once-off event or abuse by an adult, but the types of sexual abuse experienced are likely to change over the course of a lifetime. A child may, for instance, experience sexual touching by an uncle at the age of 5 , sexual harassment by peers at the age of 12 , being made to engage in sexual touching with a boyfriend at the age of 15 , and being forced to have sex with someone older than her at the age of 17 .

We therefore asked young people about how many times certain acts of sexual abuse happened during the course of their lifetime, and in the past year: in $40 \%$ of cases it occurred more than once. It is worth noting that 1 in 10 children who had experienced sexual abuse by a known adult had this experience four or more times, with approximately $30 \%$ indicating that this occurred between two and three times. Half of young people who experienced sexual abuse by another child or adolescent reported this occurring more than once. One-fifth of young people who had been sexually abused had experienced some form of sexual harassment four or more times.

The young people in this study were also asked whether they had experienced an incident of sexual abuse in the past year. The number of cases in the past year provides an estimate of how many cases agencies could expect to handle, if every case were reported to them. In the schools interviews, $13.2 \%$ of young people reported an incident of sexual abuse in the past year, which implies that if every case of sexual abuse were reported to authorities, more than one in ten children would be making such reports.

While our study could not identify causal links between one factor and another, young people who reported sexual abuse were four times more likely than those who did not to report substance misuse (though very few had been intoxicated at the time of the abuse), four times more likely to report high-risk sexual behaviour, and two to three times more likely to report post-traumatic stress disorder, anxiety or depression. Greater risk of sexual abuse was also associated with living in a rural area, school enrolment, having a flush toilet, being disabled, parental substance misuse, having poor relationships with parents, and parents who do not monitor their children's activities sufficiently.

These findings tell us that child sexual abuse is persistent over the course of children's lifetimes, and regrettably present and influential in their everyday adolescent lives. The overall prevalence rates provided here are comparatively high in relation to other available prevalence studies, but in a country where some 60000 sexual offences are reported to the police annually and with a murder rate of 17000 per annum (almost 50 murders a day), this may not be so surprising. Yet the results suggest targets for intervention, such as prevention of substance misuse, particularly among those who are parents, and providing interventions that improve parenting skills. Now that accurate national data are available, service providers and policy-makers must act to protect SA's children and break the cycle of violence. SA has committed to being a Pathfinder Country - to global leadership in the elimination of violence against children (www.end-violence.org). With these data and the growing international movement to achieve the Sustainable Development Goals (including goal 16.2, the elimination of all forms of violence against children), we hope that there is now impetus to improve the protection of SA children.

Acknowledgements. Thanks must go to the UBS Optimus Foundation, which initiated and funded the study. We also thank our reference group of SA experts who provided an invaluable sounding board throughout the study; the Optimus Study international reference group for their suggestions; and Ariane Neethling and Landon Myers for their contributions to the sampling frame. We are also indebted to our fieldwork and data capture teams, as well as to all the children, educators and parents who gave of their time to support and participate in the study.

\section{Lillian Artz}

Gender, Health, and Justice Research Unit, Faculty of Health Sciences, University of Cape Town, South Africa

\section{Catherine L Ward}

Department of Psychology, University of Cape Town, South Africa catherine.ward@uct.ac.za 


\section{Lezanne Leoschut}

Centre for Justice and Crime Prevention, Cape Town, South Africa

\section{Reshma Kassanjee}

Department of Statistical Sciences, Faculty of Science,

University of Cape Town, South Africa

\section{Patrick Burton}

Centre for Justice and Crime Prevention, Cape Town, South Africa

1. Ward CL, Artz L, Leoschut L, Kassanjee R, Burton P. Sexual violence against children in South Africa: A nationally representative cross-sectional study of prevalence and correlates. Lancet Glob Health 2018;6(4):e460-e468. https://doi.org/10.1016/S2214-109X(18)30060-3

S Afr Med J 2018;108(10):791-792. DOI:10.7196/SAMJ.2018.v108i10.13533 